Article

\title{
IGFBP2 is a potential master-regulator driving dysregulated gene network responsible for short survival in Glioblastoma multiforme
}

\author{
Manasa KP 1,2, Darius Wlochowitz' ${ }^{1}$, Edgar Wingender ${ }^{2}$, Tim Beißbarth ${ }^{1}$, Alexander Kel 2,3* \\ . Department of Medical Bioinformatics, University Medical Center Göttingen, 37099 Göttingen, Germany \\ 2. geneXplain $\mathrm{GmbH}, 38302$ Wolfenbüttel, Germany \\ 3. Institute of Chemical Biology and Fundamental Medicine SBRAS, 630090, Novosibirsk, Russia \\ * Correspondence: Alexander E. Kel: alexander.kel@genexplain.com
}

Simple Summary: The present work aims to identify molecular drivers of short survival in glioblastoma multiforme (GBM) using gene regulatory network analysis. We identified five important master regulators - IGFBP2, VEGFA, PDGFA, OSMR and AEBP1 regulating the dysregulated gene networks. Of them, IGFBP2 is found to be highly upregulated in short survivors and has established relevance in glioblastoma pathology. Further investigation on gene regulatory network revealed that FRA-1 transcription factor can be regulated by IGFBP2. FRA-1 is found to be upregulated and impacts overall survival in GBM. It is said to dysregulate at-least 50 downstream genes involved in tumor invasiveness in tumor xenografts making it an important therapeutic target for GBM intervention. We propose that IGFBP2 drives dysregulated gene network responsible for short survival in GBM.

\begin{abstract}
Only two percent of Glioblastoma multiforme (GBM) patients respond to standard care and survive beyond 36 months (long-term survivors, LTS) while the majority survives less than 12 months (short-term survivors, STS). To understand the mechanism leading to poor survival, we analyzed publicly available datasets of 113 STS and 58 LTS. This analysis revealed 198 differentially expressed genes (DEGs) that co-occur with aggressive tumor growth and may be responsible for the poor prognosis. These genes belong largely to the GO-categories "epithelial to mesenchymal transition" and "response to hypoxia". In this paper we applied upstream analysis approach which involves state-of-art promoter analysis and network analysis of the dysregulated genes potentially responsible for short survival in GBM. Transcription factors associated with GBM pathology like NANOG, NF$\kappa B$, REST, FRA-1, PPARG and seven others were found enriched in regulatory regions of the dysregulated genes. Based on network analysis, we propose novel gene regulatory network regulated by five master regulators - IGFBP2, VEGFA, VEGF165, PDGFA, AEBP1 and OSMR which can potentially act as therapeutic targets for enhancing GBM prognosis. Critical analysis of this gene regulatory network gives insights on mechanism of gene regulation by IGFBP2 via several transcription factors including the key molecule of GBM tumor invasiveness and progression FRA-1. All the observations are validated in independent cohorts and their impact on overall is studied on TCGA-GBM RNA seq data.
\end{abstract}

Keywords: Glioblastoma; master regulators; upstream analysis; IGFBP2; FRA-1; short term survivors; transcription factors 


\section{Introduction}

Glioblastoma multiforme (GBM) is the most common, highly malignant primary brain tumor ${ }^{1}$. Despite huge developments in treatment strategies, GBM poses unique treatment challenges due to tumor recurrence (34\%) and drug resistance leading to poor survival rates of less than 15 months even after advanced chemoradiotherapy ${ }^{2}$. There are as little as $2 \%$ of patients who actually respond to standard care and survive beyond 36 months (3years) ${ }^{2,3}$ clinically called as long-term survivors (LTS). Another group termed as short-term survivors (STS) are those who survive less than 12 months ${ }^{4}$. The factors that predict the long survival are not completely known.

Though several factors like age, gender, Karnofsky performance score, extent of tumor resection, radiotherapy, and chemotherapy are associated with survival and treatment responses ${ }^{5-8}$, it is evident from recent research that there are certain molecular signatures which might be driving treatment responses and thereby survival. Therefore, understanding these extreme survivor groups at molecular level may shed important light towards biological aspects driving their malignancy and survival. Promoter methylation of the gene MGMT, mutations in the genes IDH1/2 and loss of heterozygosity in chromosome $1 \mathrm{p} / 19 \mathrm{q}$ are confirmed to be highly informative about survival and treatment responses 2,3,7,9-12 . Furthermore, CHI3L1, FBLN4, EMP3, IGFBP2, IGFBP3, LGALS3, MAOB, PDPN, SERPING1 and TIMP1 gene expression is decreased in patients with long survival 10,11,13,14. Understanding these extreme survivor groups at molecular level may shed important light towards biological aspects driving their malignancy and survival.

With the advent of gene expression profiling and remarkable developments in high- throughput technologies, it is possible for us to achieve higher level molecular insights into disease biology. Databases like GEO ${ }^{15}$,Array Express ${ }^{16}$ and The Cancer Genome Atlas - TCGA ${ }^{17}$ serve as open platforms for retrieving high quality multi-omics data to find markers in cancer research. Identification of differentially expressed genes (DEGs) already serves as an important in silico strategy towards finding potential drivers of cellular state transitions. For a more refined analysis, functional annotation of genes of interest, using a priori known biological categories from the Gene Ontology ${ }^{18}$ - GO and pathway databases e.g. TRANSPATH ${ }^{\circledR}{ }^{19}, \mathrm{KEGG}^{20}$,PANTHER ${ }^{21}$ and Reactome ${ }^{22}$ has proven to be an effective hypothesis-driven approach in cancer research. Moreover, with the advent of state-of-art promoter analysis it is now possible to establish gene regulatory networks that have been used to understand the causes for gene dysregulation and identifying potential master regulators driving them. In this regard, the Genome-enhancer (my-genome-enhancer.com) incorporates an automated pipeline for such an analysis called "upstream analysis". "This strategy comprises two major steps: (1) analysis of promoters of DEGs to identify transcription factors (TFs) involved in the process under study (done with the help of the TRANSFAC ${ }^{\circledR}$ database" ${ }^{19}$ and the binding site identification algorithms, Match $^{23}$ and CMA ${ }^{24}$; (2) reconstruction of signaling "pathways that activate these TFs and identification of master regulators on the top of such pathways (done with the help of the TRANSPATH® signaling pathway database" 19 and special graph search algorithms).

In this paper we applied upstream analysis on publicly available datasets of GBM - Molecular Brain Neoplasia Data (REMBRANDT) cohort and GSE53733 to understand the gene-regulatory networks driving short-survival in GBM. This regulatory network revealed set of 12 transcription factors binding at the regulatory regions of genes of interest and 5 master regulators regulating them, namely - (a) VEGFA, mediator of angiogenesis ${ }^{25}$, promoter of stem like cells in GBM, (b) PDGF - highly amplified gene ${ }^{26}$ and key player of tumorigenesis, (c) OSMR -that orchestrates feed-forward signalling with EGFR and STAT327 to regulate tumor growth, (d) AEBP1 which has key role in pathogenesis via NF- $\kappa B$ activation ${ }^{28}$ and (e) IGFBP2. 
IGFBP2 had higher expression in STS, and was found to have an impact on overall survival as well as an established molecule of interest in GBM. IGFBP2 expression is higher in all GBM subtypes ${ }^{29}$, but is said to drive gene programs for immunosuppression in mesenchymal whereas cell proliferation in non-mesenchymal (Classical, Proneural and neural) subtypes ${ }^{29,30}$. It is said to be a marker of aggressive behavior and prognostic marker for survival ${ }^{31}$. However, the molecular mechanism by which IGFBP-2 affects disease progression and patient prognosis is not yet clear.

The current work focuses on understanding gene-regulatory networks which drive short-survival in GBM and their master regulators which can potentially act as therapeutic targets. Later, we critically discuss the role of IGFBP2 in gene-regulatory network.

\section{Results}

\subsection{Identification of differentially expressed genes}

Identifying the DEGs gives us insight on the biological semantics of a cellular state and helps to identify promising biomarkers of various disease states. The differential gene expression analysis between STS and LTS groups of GBM, from the batch corrected GSE dataset was performed using LIMMA with FDR cutoff of $5 \%$. The analysis revealed 115 significantly (adj.p-value $<0.05$ ) upregulated $(\log 2 \mathrm{FC}>0.5)$ and 83 significantly downregulated $(\log 2 \mathrm{FC}<(-0.5))$ genes. Top 5 upregulated and downregulated genes and their corresponding $\log 2 \mathrm{FC}$ are shown in Table1 and the full list is given in Table S1-A.

Table 1. The list of top 5 significantly upregulated and downregulated genes in STS identified in the GSE dataset.

\begin{tabular}{|c|c|c|c|c|}
\hline Gene Symbol & Description & $\log 2 \mathrm{FC}$ & $p$-value & adj. $p$-value \\
\hline \multicolumn{5}{|c|}{ Upregulated genes } \\
\hline CHI3L1 & Chitinase-3-like 1 & 1.370857 & 9.73E-05 & 0.0127 \\
\hline POSTN & Periostin & 1.33145 & $7.88 \mathrm{E}-07$ & 0.001627 \\
\hline PDPN & Podoplanin & 1.241589 & $6.45 \mathrm{E}-04$ & 0.028191 \\
\hline MEOX2 & $\begin{array}{c}\text { Mesenchyme homeobox } \\
2\end{array}$ & 1.159601 & 4.87E-05 & 0.010796 \\
\hline IGFBP2 & $\begin{array}{c}\text { Insulin-like growth } \\
\text { factor-binding protein } 2\end{array}$ & 1.149656 & 5.79E-05 & 0.01101 \\
\hline \multicolumn{5}{|c|}{ Downregulated genes } \\
\hline KLRC1 & $\begin{array}{l}\text { Killer cell lectin-like } \\
\text { receptor } \mathrm{C} 1\end{array}$ & -1.2187 & 3.63E-04 & 0.022188 \\
\hline KLRC2 & $\begin{array}{l}\text { Killer cell lectin-like } \\
\text { receptor } \mathrm{C} 2\end{array}$ & -1.2187 & 3.63E-04 & 0.022188 \\
\hline FUT9 & Fucosyl-transferase 9 & -1.0709 & $1.15 \mathrm{E}-04$ & 0.013921 \\
\hline DPP10 & $\begin{array}{c}\text { Dipeptidyl peptidase- } \\
\text { like } 10\end{array}$ & -1.02781 & 2.97E-05 & 0.008732 \\
\hline GABRB3 & $\begin{array}{c}\text { Gamma-aminobutyric } \\
\text { acid type A receptor } \\
\text { beta3 subunit }\end{array}$ & -0.96352 & $6.73 \mathrm{E}-05$ & 0.011057 \\
\hline
\end{tabular}

\subsection{Functional annotation of differentially expressed genes}

Functional annotation was performed to investigate biological roles of these DEGs. As shown in Figure S1(A), the top GO Biological process is extracellular structure and matrix organization with 30 DEG hits. Figure S1(B) shows the results for GO Cellular Component enrichment which revealed dysregulation of genes that belong to extracellular matrix and synaptic membrane. The important 
molecular functions enriched are channel activity and transmembrane transporter activity (Figure S1(C)). The disruption in extracellular matrix organization is one of the important signatures in glioblastoma treatment response dealing with invasiveness and malignancy ${ }^{14}$. Deeper biological insights are required in this aspect. It is interesting to see enrichment of genes known to be involved in glioma (Figure 1A). Gene signature enrichment based on hallmark gene sets of MSigDB clearly signifies the enrichment of epithelial to mesenchymal transition depicted in Figure 1B. The process of epithelial to mesenchymal transition plays a very important role in GBM survival by driving tumor invasiveness and drug resistance ${ }^{32}$. Important pathways like Aurora signaling, G2/M phase transition, TGF- $\beta$ are found to be enriched Table 2 according TRANSPATH ${ }^{\circledR}$ pathways. The full list of enrichment results can be found in Table S1-B.

(A)

(B)
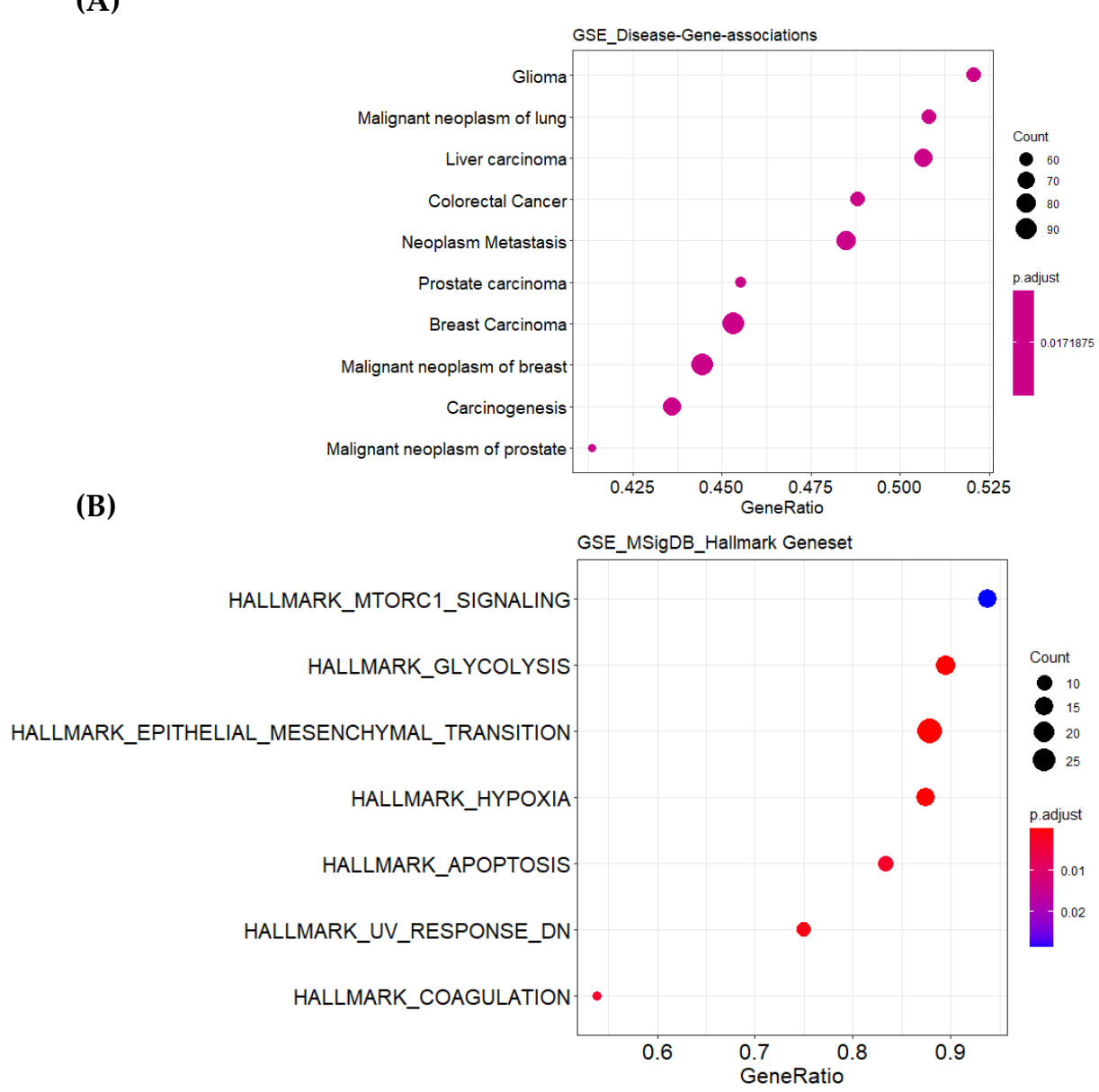

Figure 1: Functional enrichment analysis for differentially expressed genes abs (log-Fold change) of 0.5. (A) Enrichment for known Disease-gene networks in different diseases. Y-axis represents enriched ontology categories and X-axis represents GeneRatio, the percentage of all DEGs belonging to the corresponding category. The dots are sized based on gene ratio and are coloured according to their adjusted p-value. (B) Enrichment for Hallmark gene sets in Molecular signature database similar to A. 
Table 2: Transpath® Pathway (2019.3) enrichment among DEGs (STS vs. LTS)

\begin{tabular}{cccccccccc}
\hline $\begin{array}{c}\text { ID } \\
\text { (TRANSPATH) }\end{array}$ & Title & $\begin{array}{c}\text { Group } \\
\text { size }\end{array}$ & $\begin{array}{c}\text { Expected } \\
\text { hits }\end{array}$ & $\begin{array}{c}\text { Nominal P- } \\
\text { value }\end{array}$ & ES & $\begin{array}{c}\text { Rank at } \\
\text { max }\end{array}$ & NES & FDR & $\begin{array}{c}\text { Number of } \\
\text { hits }\end{array}$ \\
\hline CH000001004 & $\begin{array}{c}\text { Aurora-A cell } \\
\text { cycle regulation }\end{array}$ & 68 & 67.26247 & 0 & 0.42261 & 8347 & 4.13803 & 0 & 68 \\
\hline CH000000919 & $\begin{array}{c}\text { Cyclosome } \\
\text { regulatory } \\
\text { network }\end{array}$ & 77 & 76.16486 & 0 & 0.348952 & 7336 & 3.728426 & 0 & 77 \\
\hline CH000000694 & $\begin{array}{c}\text { G2/M phase } \\
\text { (cyclin B: Cdk1) }\end{array}$ & 66 & 65.28416 & 0 & 0.374627 & 6641 & 3.587532 & 0 & 66 \\
\hline CH000000879 & Caspase network & 83 & 82.09978 & 0 & 0.333518 & 8414 & 3.523174 & 0 & 83 \\
\hline CH000000711 & TGFbeta pathway & 153 & 151.3406 & 0 & 0.2327 & 8431 & 3.346102 & 0 & 151 \\
\hline
\end{tabular}

\subsection{Identifying the master regulators of dysregulated gene networks}

Reconstruction of the disease-specific regulatory networks can help identify potential master regulators which may serve as therapeutic targets to block a specific pathological regulatory cascade. Using the gene regulatory analysis as a first step we analyzed enrichment of transcription factor binding sites in promoters of upregulated genes of STS using DNA-binding motifs listed in the TRANSFAC® library. 274 transcription factors (Table S1-C) enriched for CCKR signaling, interleukin signaling, PDGF signaling, WNT signaling were found to have their binding sites enriched, full enrichment results can be found in Table S1-D.

Next, we applied the Composite Module Analyst (CMA) and identified-two modules involving 12 transcription factor binding site combinations that regulate the expression of genes of interest. CMA revealed the following modules comprising clustering binding sites for the following TFs: Module1: HNF3B, NANOG, NFKAPPAB, TAF1, TCF4, FRA1; Module2: PPARG, TAL1, REST, POU6F1, FOSJUN \& PBX. The modules, transcription factors and their significance are depicted in Figure S2. Differential expression statistics for the 12 transcription factors are given in Table S2. Among them, FRA-1 transcription factor was found to be $p$-value significant and upregulated in short-term survivors of GBM $(\log 2 F C=0.023$, p-value $=0.008$, adj.p-value $=0.093)$ Table S2

Finally, we reconstructed the signaling network that activates the TFs revealed by CMA analysis and thereby identifying the top regulators in these networks using TRANSPATH database. The process identified 5 important master regulators that are plausible drivers of short survival in GBM: IGFBP2, VEGFA/VEGF165, PDGFA, AEBP1 and OSMR. All the master regulators were found to be significantly upregulated in short-term survivors. The regulatory network reconstructed along with six master regulators is shown in Figure 2, the master regulators and their LogFC in STS are listed in Table 3. Since VEGF165 is a splice variant of VEGF-A, only the latter will be considered further on. 


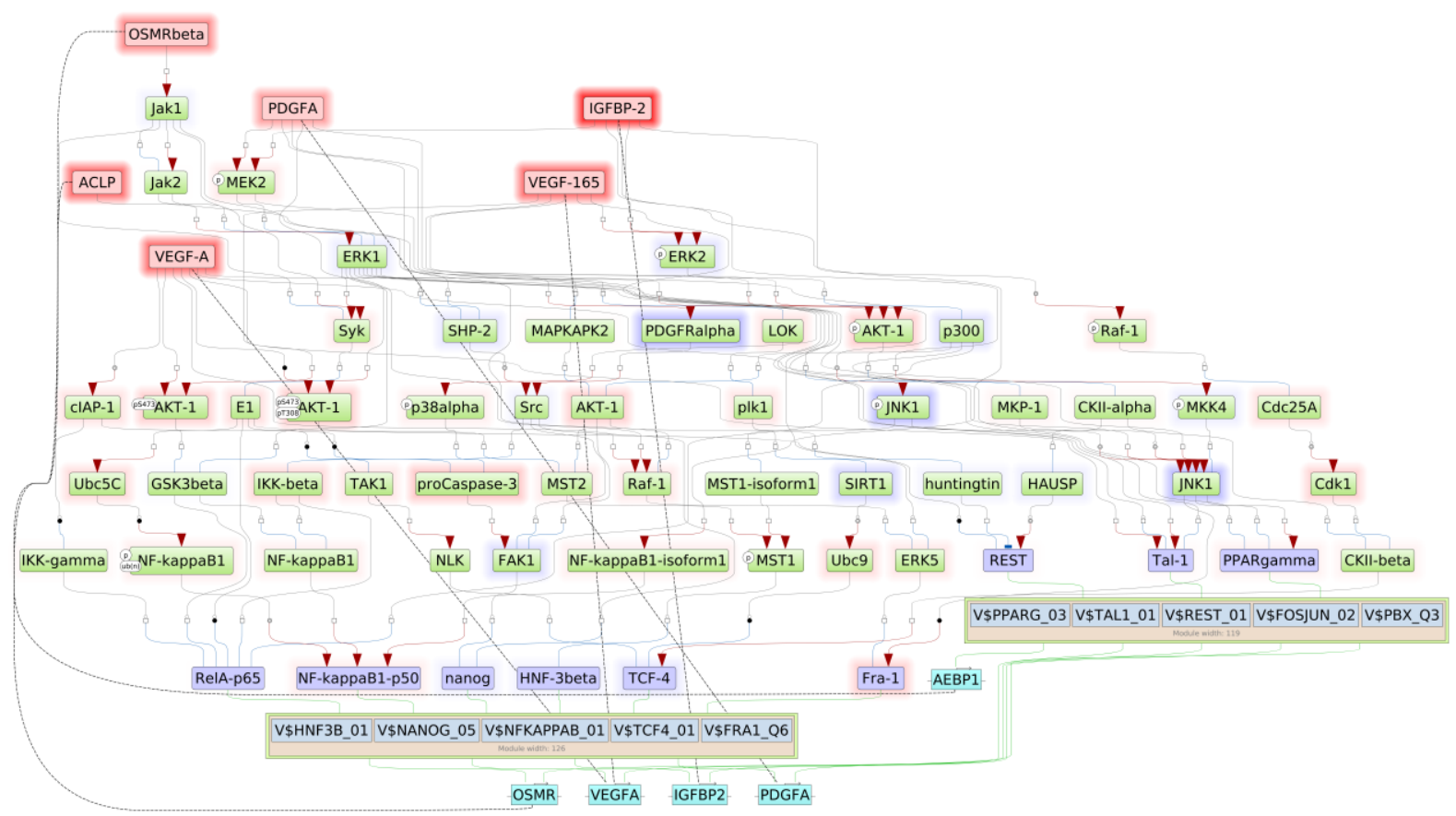

Figure 2. Gene regulatory network of six master regulators (red nodes) regulating two transcription factor modules (purple nodes) enriched in promoters of highly upregulated genes of STS. The dotted lines from genes to such signalling proteins represents the transcription and translation processes. The outside box filling is based on $\operatorname{LogFC}$ and are filled red when upregulated (Log2FC $>0.2 \&$ p-value $<0.05)$ and filled blue when downregulated $(\log 2 \mathrm{FC}<0.2 \&$ p-value $<0.05)$ in the current study ${ }^{68}$.

Table 3. Table of the master regulators identified, their description, LogFC in STS and number of TFs regulated.

\begin{tabular}{ccccc}
\hline $\begin{array}{c}\text { Molecule } \\
\text { Name }\end{array}$ & Gene Description & $\begin{array}{c}\text { HGNC Gene } \\
\text { symbol }\end{array}$ & LogFC in STS & $\begin{array}{c}\text { No. of TFs } \\
\text { regulated }\end{array}$ \\
\hline IGFBP-2 & $\begin{array}{c}\text { Insulin like growth factor binding } \\
\text { protein 2 }\end{array}$ & IGFBP2 & 1.12 & 9 \\
\hline ACLP & AE binding protein 1 & AEBP1 & 0.7829 & 9 \\
\hline VEGF-A & vascular endothelial growth factor A & VEGFA & 0.77833 & 9 \\
\hline VEGF-165 & vascular endothelial growth factor A & VEGFA & 0.77833 & 9 \\
\hline OSMRbeta & Oncostatin M receptor & OSMR & 0.6345 & 8 \\
\hline PDGF-A & platelet derived growth factor subunit & PDGFA & 0.52932 & 9 \\
\hline & A & & & \\
\hline
\end{tabular}

\subsection{Validating the expression of master regulators in other cohorts}

Expression patterns of the above identified master regulators have been validated in two different cohorts. A) TCGA-GBM microarray data and B) GSE16011. The expression patterns were similar and there is a significant upregulation of all master regulators except for VEGFA (GSE16011: adj_pvalue $=0.069$, TCGA-GBM: adj_pvalue $=0.075$ ) Table S1-E \& S1-F. The differential expression values are given in Table 4. 
Table 4. Expression of master regulators across survival groups (STS and LTS resp,) and across 3 datasets - GSE, TCGA-GBM microarray and GSE16011.

\begin{tabular}{ccccccc}
\hline $\begin{array}{c}\text { Master } \\
\text { Regulator }\end{array}$ & \multicolumn{2}{c}{ GSE } & \multicolumn{2}{c}{ GSE16011 } \\
\hline & $\begin{array}{c}\text { Log2FC (ST vs } \\
\text { LT) }\end{array}$ & $\begin{array}{c}\text { adj.p- } \\
\text { value }\end{array}$ & $\begin{array}{c}\text { Log2FC (ST vs } \\
\text { LT) }\end{array}$ & $\begin{array}{c}\text { adj.p- } \\
\text { value }\end{array}$ & $\begin{array}{c}\text { Log2FC (ST vs } \\
\text { LT) }\end{array}$ & $\begin{array}{c}\text { TCGA } \\
\text { adj.p- } \\
\text { value }\end{array}$ \\
\hline IGFBP2 & 1.149656 & $4.87 \mathrm{E}-05$ & 2.03 & $4.598 \mathrm{E}-04$ & 1.098383 & $5.00 \mathrm{E}-06$ \\
AEBP1 & 0.782898 & $7.75 \mathrm{E}-05$ & 1.723 & 0.00102 & 0.971786 & $3.96 \mathrm{E}-06$ \\
PDGFA & 0.529317 & $4.55 \mathrm{E}-04$ & 1.68005 & $4.709 \mathrm{E}-09$ & 0.825137 & $2.07 \mathrm{E}-05$ \\
VEGFA & 0.778333 & $5.20 \mathrm{E}-04$ & 0.88 & 0.06904 & 0.50068 & 0.07529 \\
OSMR & 0.634496 & $8.65 \mathrm{E}-04$ & 1.9577 & $4.24 \mathrm{E}-05$ & 0.48667 & 0.03189 \\
\hline
\end{tabular}

\subsection{Validating the master regulators in TCGA-GBM cohort}

The TCGA-GBM microarray data containing 258 STS and 49LTS is used to validate the above identified drivers of short survival. The data is pre-processed and differential gene expression analysis is performed Figure S3. Same cut-offs for Log2FC and adj.p-value are used. We identified 171 genes upregulated in STS of GBM (Log2FC > $0.5 \&$ adj.p-val <0.05) full list in Table S1-E. 49 of them were in common between GSE dataset and TCGA-GBM, full differential gene expression analysis result given in Table S1-G. Hence, the transcription factors with enriched binding sites across the 2 datasets were expected to vary. We identified set of 16 transcription factors Table S3 and 12 master regulators upstream Table S4 of them regulating the gene-network in STS.

As a result, the TCGA-GBM dataset validates IGFBP2, AEBP1(ACLP) and PDGFA as master regulators driving dysregulated gene network in STS. We also found that binding sites for FRA-1 transcription factor are statistically significantly enriched at the regulatory regions of dysregulated genes including IGFBP2 even in TCGA-GBM cohort Table S5. FRA-1 binding sites on IGFBP2 regulatory region in TCGA-cohort is depicted in Figure 3.

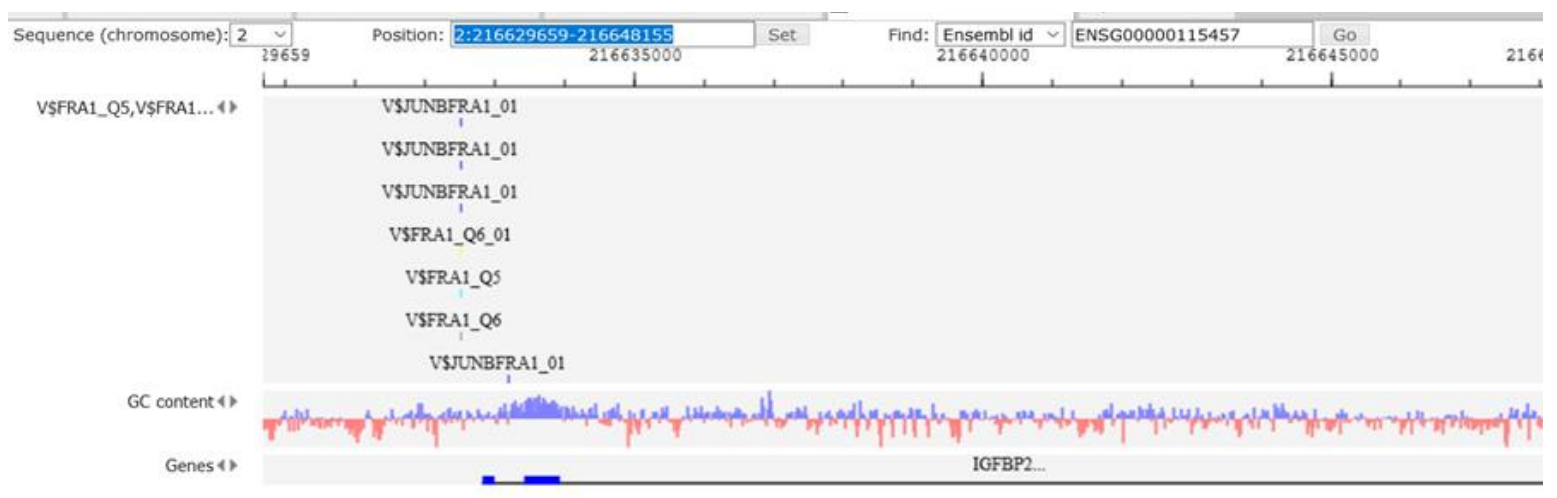

Figure 3. Regulation of IGFBP2 by FRA-1/FOSL1 Transcription factor. FRA-1 binding sites on promoter of IGFBP2 master regulator as observed in TCGA-GBM validation cohort is given.

\subsection{Impact of master regulators on survival in $G B M$}

Univariate survival analysis was used to study the impact of these master regulators and their regulated transcription factors on overall survival in GBM. Patients are split into non-overlapping $\mathbf{5 0} \%$ upper 
and lower quantiles. All master regulators were found to have significant impact upon survival except VEGFA. Additionally, Fra-1 transcription factor, also known as Fos-related antigen 1 (FOSL1), was found to be significant Figure 4.
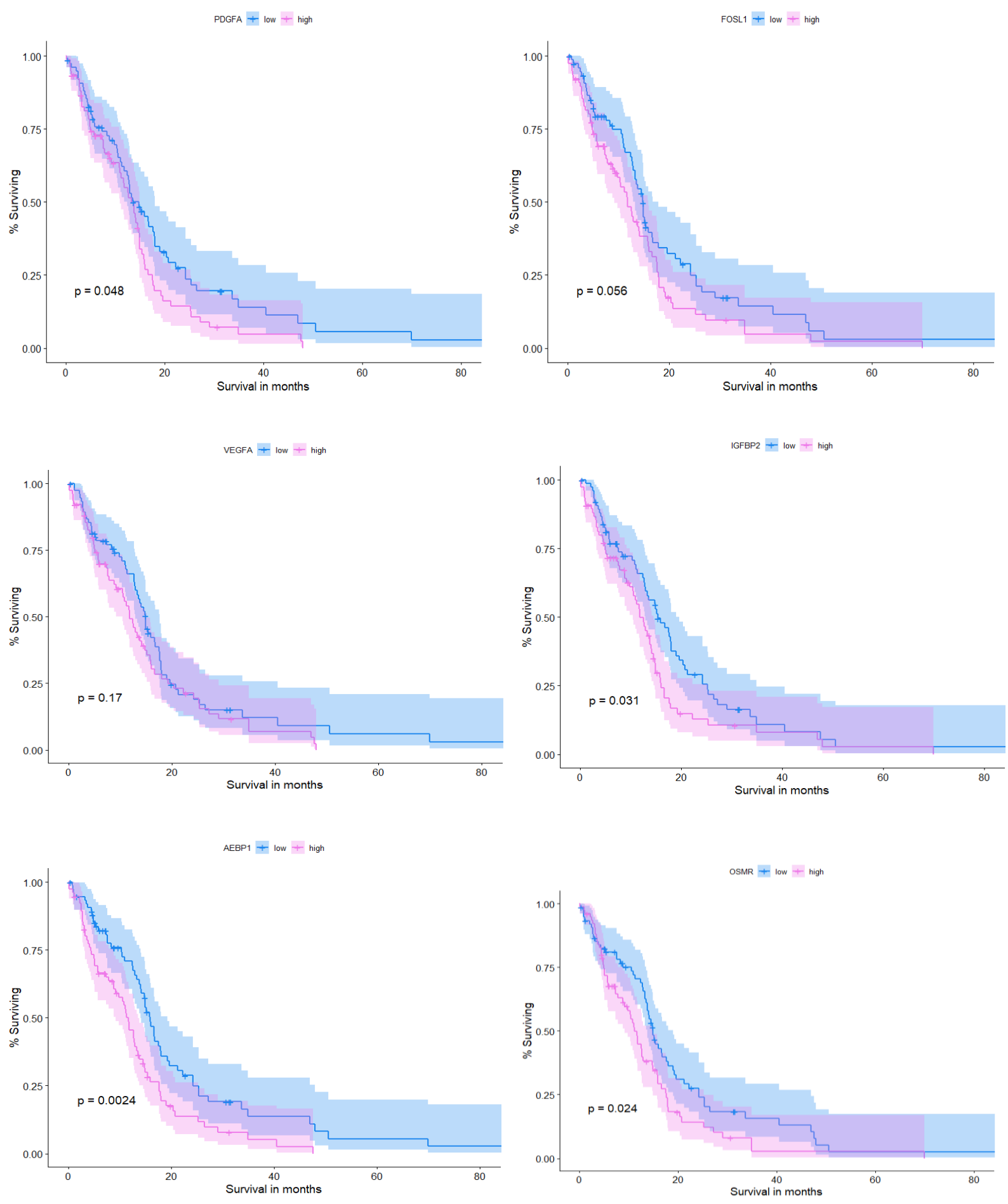

Figure 4. Univariate survival analysis of 152 RNA sequencing data of TCGA GBM cohort is studied using Survival and Survminer packages in R. Out of five master regulators, all but VEGFA (AEBP1, OSMR, PDGFA and IGFBP2) had a statistically significant impact on survival based on 50\% upper and lower quantiles (P-value cut-off of 0.05). Out of the two modules comprising 12 transcription factors, FRA-1/FOSL1 transcription factor was found to have impact on survival. 


\subsection{Master regulator expression patterns across GBM Subtypes}

Based on regulatory landscape of GBM, there are 4 subtypes - Classical, Mesenchymal, Proneural and Neural 83. There is a significant level of intertumoral as well as intra-tumoral heterogeneity within each of them ${ }^{83,84}$. We looked for expression patterns Figure S4, of above identified master regulators in each subtype as well as tried identifying differentially expressed genes between STS and LTS Table S6 within each subtype Supplementary file 3 . None of the master regulators were found to be significantly differentially expressed between survivor groups in any subtypes.

\section{Discussion}

Gene regulatory networks represent the causal regulatory relationships between transcription factors (TFs) and their gene targets, which enables us to discover dysregulated genes in certain biological states $^{33}$. Comparative studies of short-term survivors and long-term survivors of GBM showed that gene-expression programs executed across survival groups vary significantly. In the light of these findings, we sought to apply an upstream analysis approach to gain insight about gene regulatory networks driving short survival.

In the promoter analysis, we identified a set of $12 \mathrm{TFs}$ that are enriched and binding to the regulatory regions of dysregulated genes in short term survivors (upregulated in STS). The TFs NANOG and REST have been said to be critical for self-renewal and maintenance of oncogenic signatures in glioblastoma stem-like cells ${ }^{34,35}$, PPARG has emerged as a promising therapeutic target as its agonists increased median survival in GBM patients ${ }^{36}$, NF- $\kappa B$ is implicated in several processes like invasion, epithelialmesenchymal transition ${ }^{37}$, resistance to radiotherapy ${ }^{38}$ and maintenance of cancer stem-like cells ${ }^{39}$, FRA1/FOSL1 has been reported to be important in maintenance/progression of malignant glioma ${ }^{40}$. Among these 12 TFs, we found that FRA-1 has a significant impact upon survival and has higher expression in STS. Debinski et al., (2005) ${ }^{40}$ hypothesized that any AP1 stimulating signals like epidermal growth factor (EGF), leukemia inhibitory factor, Oncostatin M, FGF-2 can positively regulate FRA-1. VEGF-D was shown to be regulated by FRA-1 and is a known prognostic factor in other aggressive cancers 41,42

Graph analysis of the signal transduction network upstream of these transcription factors identified five potential master-regulators that might explain gene dysregulation in STS, namely - insulin like growth factor binding protein (IGFBP2), vascular endothelial growth factor A(VEGF-A), its isoform VEGF165, platelet Derived growth Factor A(PDGFA), Oncostatin M(OSMR) and Adipocyte Enhancer binding protein (AEBP1).All the identified master regulators were upregulated in STS and their expression patterns were validated in 2 other independent cohorts. We found that all master regulators except VEGFA has an impact on overall survival in the GBM patients. IGFBP2, AEBP1 and PDGFA master regulators driving short survival were validated in TCGA-GBM cohort. Out of them, IGFBP2 had higher expression in STS. The insulin-like growth factor binding protein 2 (IGFBP2) is said to be one of most potential glioma oncogene and functions as a hub of oncogenic signalling pathways by regulating pro-tumorigenic signals of tumor initiation and progression. It is established that Exogenous IGFBP2 promotes proliferation, invasion, and chemoresistance to temozolomide in glioma cells via integrin $\beta 1$ by promoting ERK phosphorylation and nuclear translocation ${ }^{43,44}$.IGFBP2 is considered as one of the strongest biomarkers of aggressive behavior in $\mathrm{GBM}^{29,45}$ and also a prognostic marker for survival 29,46 .

Here, we propose that IGFBP2 can be a potential regulator of FRA-1 transcription factor. IGFBP2 induced RAF/MAPK signalling (Figure 2) activates FRA-1. It is earlier shown that IGFBP2 and FRA-1 regulated transcription of VEGF $41,42,47$, which is the second most dysregulated master regulator in our network. We find that promoters of the genes of all the five master regulators reported in the study have binding sites for FRA-1 transcription factor. It is reported that enhanced ERK signalling, that may be triggered by these master-regulators, may lead to mitogen-induced FRA- 1 transcription ${ }^{48}$ as well as 
its protection from proteasomal degradation ${ }^{49}$. As per the gene regulatory network developed, it is possible that FRA-1 mediates the positive feedback loop where FRA-1 transcribes master regulator genes in cooperation with other TFs and master regulator gene products enhance/potentiate FRA-1. Figure 5 combines prior knowledge in the field and gene-regulatory network developed in our study (Figure 2,5).

In summary, the current work proposes a gene regulatory network in STS regulated by five master regulators - IGFBP2, VEGFA, PDGFA, OSMR and AEBP1 which can potentially act as biomarkers of GBM prognosis and as therapeutic targets for enhancing GBM prognosis. The work also proposes a novel mechanism of gene dysregulation by IGFBP2 by modulating a key molecule of tumor invasiveness and progression - FRA-1 transcription factor. All the genes encoding these five master regulators have binding sites for FRA-1 in their promoters. FRA-1 and the master regulators are in a positive feedback loop to orchestrate a complex tumorigenic program leading to poor survival in GBM.

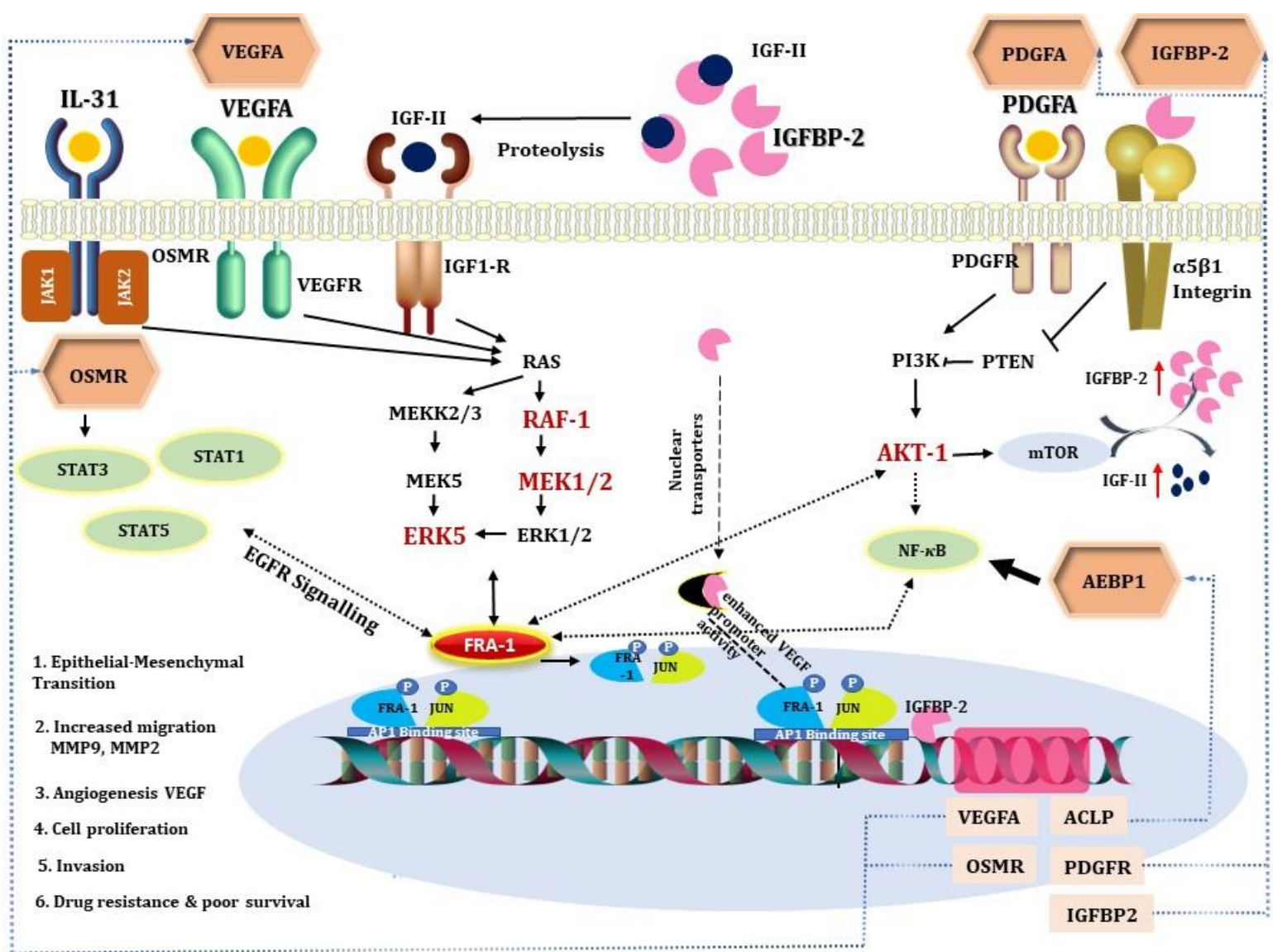

Figure 5: Diagram combining prior knowledge about of role of IGFBP2 in GBM and the gene-regulatory network developed in the study. The hexagons are master regulators identified in our analysis. All the intermediates of the gene regulatory network are colored red if upregulated in STS, and black if not present in the network. Dotted black line if the knowledge is through literature, continuous black line if known through gene regulatory network. Blue dotted lines represent gene regulatory connections between master regulators and their corresponding genes transcribed by target transcription factors.

VEGFA, PDGFA, IGFs and IL-31 activate RAF/MEK/ERK signalling which mediates cell survival through PI3K-AKT pathway ${ }^{50,51}$. MEK2/RAF1/ERK5 and AKT-1 are found to be upregulated in STS, suggestive of activated ERK signalling which can contribute to drug resistance ${ }^{52,53}$. IGFBP2 activates IGFR either by increasing bioavailability of IGFs or by direct interaction with its functional domain. Integrin acts as receptor for IGFBP2 extracellular signals ${ }^{43,44}$ and modulates NF- $\kappa B$ signalling. IGFBP2 by nuclear translocation 47 is involved in transcriptional regulation of the 
VEGF gene and modulates angiogenesis ${ }^{47}$. STAT3 and NF- $\kappa B$ are said to be the two major downstream transcription factors of IGFBP2 that direct tumorigenic intracellular signalling 55 via EGFR signalling. . Oncostatin $M$, receptor for cytokine IL31 is a regulator of EGFR signalling27. All the 5 master regulators have binding sites for FRA-1. In the figure we depicted the possible positive feedback loop between FRA-1 and the master regulators to orchestrate complex tumorigenic program of invasiveness, migration, drug resistance and angiogenesis.

\section{Materials and Methods}

\subsection{Data Collection}

The genome wide expression profiles based on Human Genome U133 plus 2.0 array and clinical information of patients with GBM were collected from public repository of GEO database GSE10847456(https://www.ncbi.nlm.nih.gov/geo/query/acc.cgi?acc=GSE108474) and GSE5373312 (https://www.ncbi.nlm.nih.gov/geo/query/acc.cgi?acc=GSE53733). The 2 datasets were pooled together leading to 113 and 58 samples corresponding to short-term survivors (STS; survival < 12 months) and long-term survivors (LTS; survival > 36 months) with GBM, respectively Table 5.

Table 5. Statistics of datasets studied in this work. The datasets with labels GSE' were collected from GEO database.

\begin{tabular}{cccc}
\hline Platform & $\begin{array}{c}\text { Short-term } \\
\text { survivors }\end{array}$ & $\begin{array}{c}\text { Long-term } \\
\text { survivors }\end{array}$ \\
\hline GSE53733 ${ }^{12}$ & $\begin{array}{c}\text { HU133 plus } \\
2.0 \text { arrays }\end{array}$ & 16 & 23 \\
\hline GSE10847456 & HU133 plus & & \\
& 2.0 arrays & 97 & 35 \\
\hline
\end{tabular}

\subsection{Affymetrix microarray data pre-processing}

The raw data files (. CEL format) for GSE108474 and GSE53733 were collected from GEO databasefrom here on called as GSE dataset. RMA algorithm is used in R (affy package) for background correction, quality check and normalization to obtain $\log 2$ transformed expression values ${ }^{57}$. Batch correction of the pooled expression data was performed using empirical Bayes framework is performed 58. This batch corrected file is used for further analysis. Multiple Affymetrix ids were summarized to genes ids by choosing the maximum out of probe intensities of multiple probes belonging to single gene. The final expression matrix comprised 21526 probes and 171 samples.

\section{Differential gene expression (DEG) analysis}

LIMMA (Linear Models for Microarray Data) method was applied to identify differentially expressed genes ${ }^{59}$. It is an efficient tool which is stable even for experiments with small samples. Differential gene expression analysis of 171 samples of GSE dataset was performed with BenjaminiHochberg adjusted P_Value. Only genes with $\mid \log 2$ fold change (FC) $\mid \geq 0.5$ and adjusted P values < 0.05 were selected as the DEGs for subsequent analysis.

\subsection{Databases used in the study}

Transcription factor binding sites in promoters and enhancers of differentially expressed genes were analyzed using known DNA-binding motifs described in the TRANSFAC ${ }^{\text {library, }}$, release 2019.3 (geneXplain GmbH, Wolfenbüttel, Germany) (https://genexplain.com/transfac)60. The master regulator search uses the TRANSPATH® database (BIOBASE), release 2019.3 (geneXplain GmbH, Wolfenbüttel, 
Germany) (https://genexplain.com/transpath) ${ }^{19}$. A comprehensive signal transduction network of human cells is built by the software based on reactions annotated in TRANSPATH®. The information about drugs corresponding to identified drug targets and clinical trials references were extracted from HumanPSD $^{\mathrm{TM}}$ database $^{61}$, release 2020.2 (https://genexplain.com/humanpsd). The Ensembl ${ }^{62}$ database release Human99.38 (hg38) (http://www.ensembl.org) was used for gene IDs representation and Gene Ontology ${ }^{18}(\mathrm{GO})$ (http://geneontology.org) was used for functional classification of the studied gene set.

\subsection{Functional Annotation}

To explore the biological importance of DEGs, gene set enrichment analysis is performed. GSEA is an efficient method to determine whether the genes of interest show statistically significant enrichment between different biological states. Gene ontology enrichments for cellular component, biological process and molecular functions were performed. To investigate the top enriched ontology terms 1000 random permutations were done and adjusted p-value cutoff of 0.05 is used. The dysregulated gene networks enrichment also gives useful insight about known disease signatures ${ }^{63}$. The hallmark gene set of MsigDB ${ }^{64}$ defines specific biological states or processes. Enrichment analysis is performed in $\mathrm{R}$ using DOSE ${ }^{65}$ package. PANTHER pathway enrichment of the identified transcription factors was performed using EnrichR tool ${ }^{66}$. TRANSPATH ${ }^{19}$ pathway enrichment was performed using geneXplain platform.

\subsection{Genome Enhancer pipeline}

The approaches mentioned above helps us in understanding the impact of the differentially expressed genes in GBM biology. To understand the reason behind this dysregulation, Genome enhancer pipeline of geneXplain is used (my-genome-enhancer.com). Significantly upregulated genes in STS were used in this workflow.

It works in 2 steps.

\section{A. Analysis of enriched transcription factor binding sites and composite modules}

Binding of transcription factors to the transcription factor binding sites in promoters and like enhancers is key to mediation of transcriptional regulation of genes. Classically, enhancers are defined as regions in the genome that increase transcription of one or several genes when inserted in either orientation at various distances upstream or downstream of the gene ${ }^{24}$. Enhancers typically have a length of several hundreds of nucleotides and are bound by multiple transcription factors ${ }^{19}$.

Identifying such clusters of binding sites for such transcription factors (composite-modules) that act as potential condition-specific enhancers of the target genes in their upstream regulatory regions ($1000 \mathrm{bp}$ upstream of transcription start site (TSS)) and the transcription factors which regulate the genes through such enhancers is a determining step to understand regulatory mechanism. PANTHER pathway enrichment of the above identified transcription factors were identified using EnrichR tool ${ }^{66}$.

We use Composite Module Analyst (CMA) ${ }^{24}$ method to detect such potential enhancers, as targets of multiple TFs bound in a to the regulatory regions of the genes of interest. CMA applies a genetic algorithm to construct a generalized model of the enhancers by specifying combinations of TF motifs (from TRANSFAC ${ }^{\circledR}$ ) whose sites are most frequently clustered together in the regulatory regions of the studied genes. The transcription factors are ranked based on (a)The Yes-No ratio between frequencies of yes sequences versus No sequences and gives level of enrichment of indicated transcription factor (b) Regulatory score, which is a measure of involvement of a transcription factor in controlling expression of genes that encode master regulators. CMA identifies the transcription factors that through their cooperation provide a synergistic effect and thus have a great influence on the gene regulation process. 


\section{B. Finding master regulators in networks}

The second step involves the signal transduction database TRANSPATH® and special graph search algorithms to identify common regulators of the revealed transcription factors. These master regulators appear to be the key candidates for therapeutic targets as they have a master effect on regulation of intracellular pathways that activate the pathological process of our study. Master regulators regulating the above revealed transcription factors are not only ranked (a) based on LogFC but also (b)CMA score - which signifies how strong is the potential for this gene to be regulated by transcription factors of interest (c) Master regulator score - which signifies how strong is the potential of this gene product to regulate activity of those transcription factors. Selected master regulators can also be visualized and with possibility to map the $\log F C, p$ value on the created regulatory network.

\subsection{Validation of observed gene signatures}

The raw TCGA HT-Hg_U133 microarray data of 560 GBM samples were downloaded from TCGA legacy archive. The GSE16011 raw. CEL data was downloaded from GEO repository. Both raw datasets were processed and analyzed independently following same steps as mentioned earlier. These two datasets are used to observe and validate the expression pattern of master regulators across the two survival groups (see Table 6). GSE16011 comprises of data generated at a single center and is used in several studies ${ }^{62-64}$, unlike TCGA. TCGA-GBM microarray data PCA plots are given Figure S3 and no significant batch effects in the context of survival groups were found.

Table 6. Statistics of two validation datasets.

\begin{tabular}{cccc}
\hline Datasets & Platform & $\begin{array}{c}\text { Short-term } \\
\text { survivors }\end{array}$ & $\begin{array}{c}\text { Long-term } \\
\text { survivors }\end{array}$ \\
\hline GSE1601167 & $\begin{array}{c}\text { HU133 plus } \\
2.0 \text { arrays }\end{array}$ & 93 & 16 \\
\hline $\begin{array}{l}\text { TCGA-GBM } \\
\text { microarray }\end{array}$ & HU133 & 271 & 49 \\
\hline
\end{tabular}

\subsection{Validation of Master regulators}

The TCGA-GBM microarray data downloaded from TCGA legacy archive is processed in same fashion as GSE. Similar cutoffs (Log2FC, p-value) and parameters are used to identify enriched transcription factors and network analysis in-order to understand drivers of gene-regulatory networks in short survival.

\subsection{Impact on survival}

Master regulators and their target transcription factors affect the whole regulatory network and therefore can have an independent impact on survival in GBM patients. Tier 3 RNA sequencing data and clinical data for 152 TCGA GBM cohort is downloaded from TCGA-GBM project. Survival and Survminer libraries in $\mathrm{R}$ are used to perform univariate survival analysis. Univariate survival analysis is used to understand the impact of individual master regulator on survival in GBM with nonoverlapping $50 \%$ upper and lower quantiles.

\section{Conclusion}

In the work presented, we have identified candidate master regulators responsible for gene dysregulation in short-term survivors. These candidates have sufficient experimental evidence towards their role in GBM. Out of reported five master regulators, IGFBP2 is established as the most promising 
master regulator. Through the gene regulatory network analysis, we also propose that FRA-1 is a plausible downstream regulator of IGFBP2-induced signalling in short-term survivors of GBM.

Funding: This project has received funding from the European Union's Horizon 2020 research and innovation programme under the Marie Skłodowska-Curie grant agreement No 766069.

Availability of data and materials: The datasets analyzed during the current study are available from the previous publications ${ }^{60,61}$. The results of the analysis performed with the means of the pipeline My-Genome-Enhancer in geneXplain platform are available at the following URL 68 https://platform.genexplain.com/bioumlweb/\#anonymous=true\&de=data/Projects/Regulatory_networks_of_Glio blastoma_survival/Data/GSE_dataset/Master_regulator_network

- All tables and diagrams are available at the respective folders in the geneXplain platform. Data are also available in the Additional files provided in the Supplement to the publication.

Conflicts of Interest: The authors Manasa KP, Darius Wlochowitz and Tim Beißbarth are from Department of Medical Bioinformatics, University Medical Center Göttingen, Manasa KP, Alexander Kel and Edgar Wingender are employees of geneXplain $\mathrm{GmbH}$, which maintains and distributes the geneXplain platform and My-GenomeEngancer.com used in this study.

Acknowledgement: We thank the reviewers for their valuable suggestions to improve the manuscript

\section{References}

1. Wen, P. Y. \& Kesari, S. Malignant gliomas in adults. New England Journal of Medicine vol. 359 492-507 (2008).

2. Krex, D. et al. Long-term survival with glioblastoma multiforme. Brain 130, 2596-2606 (2007).

3. Das, P. et al. A clinicopathological and molecular analysis of glioblastoma multiforme with longterm survival. J. Clin. Neurosci. 18, 66-70 (2011).

4. Shinawi, T. et al. DNA methylation profiles of long- and short-term glioblastoma survivors. Epigenetics 8, 149-156 (2013).

5. Sonoda, Y. et al. Long-term survivors of glioblastoma: Clinical features and molecular analysis. Acta Neurochir. (Wien). 151, 1349-1358 (2009).

6. Scott, J. N. et al. Which glioblastoma multiforme patient will become a long-term survivor? A population-based study. Ann. Neurol. 46, 183-188 (1999).

7. Zhang, X., Zhang, W., Cao, W. D., Cheng, G. \& Zhang, Y. Q. Glioblastoma multiforme: Molecular characterization and current treatment strategy (Review). Experimental and Therapeutic Medicine vol. 3 9-14 (2012).

8. Lee, Y. et al. Gene expression analysis of glioblastomas identifies the major molecular basis for the prognostic benefit of younger age. BMC Med. Genomics 1, 52 (2008).

9. Chen, J. R., Yao, Y., Xu, H. Z. \& Qin, Z. Y. Isocitrate dehydrogenase (IDH)1/2 mutations as prognostic markers in patients with glioblastomas. Med. (United States) 95, (2016).

10. Franceschi, S. et al. Investigating molecular alterations to profile short- and long-term recurrence-free survival in patients with primary glioblastoma. Oncol. Lett. 10, 3599-3606 (2015).

11. Han, S., Meng, L., Han, S., Wang, Y. \& Wu, A. Plasma IGFBP-2 Levels after Postoperative Combined Radiotherapy and Chemotherapy Predict Prognosis in Elderly Glioblastoma Patients. PLoS One 9, e93791 (2014). 
12. Reifenberger, G. et al. Molecular characterization of long-term survivors of glioblastoma using genome- and transcriptome-wide profiling. Int. J. Cancer 135, 1822-1831 (2014).

13. Bi, W. L. \& Beroukhim, R. Beating the odds: Extreme long-term survival with glioblastoma. Neuro-Oncology vol. 16 1159-1160 (2014).

14. De Vega, S., Iwamoto, T. \& Yamada, Y. Fibulins: Multiple roles in matrix structures and tissue functions. Cellular and Molecular Life Sciences vol. 66 1890-1902 (2009).

15. Barrett, T. et al. NCBI GEO: Archive for functional genomics data sets - Update. Nucleic Acids Res. 41, D991-D995 (2013).

16. Athar, A. et al. ArrayExpress update - From bulk to single-cell expression data. Nucleic Acids Res. 47, D711-D715 (2019).

17. Grossman, R. L. et al. Toward a Shared Vision for Cancer Genomic Data. N. Engl. J. Med. 375, 1109-1112 (2016).

18. Ashburner, M. et al. Gene ontology: Tool for the unification of biology. Nature Genetics vol. 25 25-29 (2000).

19. Krull, M. et al. TRANSPATH®: An integrated database on signal transduction and a tool for array analysis. Nucleic Acids Research vol. 31 97-100 (2003).

20. Kanehisa, M., Furumichi, M., Sato, Y., Ishiguro-Watanabe, M. \& Tanabe, M. KEGG: integrating viruses and cellular organisms. Nucleic Acids Res. (2020) doi:10.1093/nar/gkaa970.

21. Thomas, P. D. et al. PANTHER: a library of protein families and subfamilies indexed by function. Genome Res. (2003).

22. Jassal, B. et al. The reactome pathway knowledgebase. Nucleic Acids Res. 48, D498-D503 (2020).

23. Kel, A. E. et al. MATCH ${ }^{\mathrm{TM}}$ : A tool for searching transcription factor binding sites in DNA sequences. Nucleic Acids Res. 31, 3576-3579 (2003).

24. Waleev, T. et al. Composite Module Analyst: Identification of transcription factor binding site combinations using genetic algorithm. Nucleic Acids Res. 34, W541 (2006).

25. Xu, C., Wu, X. \& Zhu, J. VEGF promotes proliferation of human glioblastoma multiforme stemlike cells through VEGF receptor 2. Sci. World J. 2013, (2013).

26. Martinho, O. \& Reis, R. M. Malignant Gliomas: Role of Platelet-Derived Growth Factor Receptor A (PDGFRA). in Tumors of the Central Nervous System, Volume 1 109-118 (Springer Netherlands, 2011). doi:10.1007/978-94-007-0344-5_12.

27. Jahani-As, A. et al. Control of glioblastoma tumorigenesis by feed-forward cytokine signaling. Nat. Neurosci. 19, 798-806 (2016).

28. Majdalawieh, A. F., Massri, M. \& Ro, H. S. AEBP1 is a Novel Oncogene: Mechanisms of Action and Signaling Pathways. Journal of Oncology vol. 2020 (2020).

29. Phillips, L. M. et al. Glioma progression is mediated by an addiction to aberrant IGFBP2 expression and can be blocked using anti-IGFBP2 strategies. J. Pathol. 239, 355-364 (2016).

30. Cai, J. et al. Immune heterogeneity and clinicopathologic characterization of IGFBP2 in 2447 glioma samples. Oncoimmunology 7, e1426516 (2018).

31. Lindström, M. S. Expanding the scope of candidate prognostic marker IGFBP2 in glioblastoma. Bioscience Reports vol. 39 (2019). 
32. Iwadate, Y. Epithelial-mesenchymal transition in glioblastoma progression. Oncology Letters vol. 11 1615-1620 (2016).

33. Marbach, D. \& Stolovitzky, G. Robert Küffner 6,11, Nicole M Vega 3-5. Robert J Prill 11, (2012).

34. Bradshaw, A. et al. Cancer Stem Cell Hierarchy in Glioblastoma Multiforme. Frontiers in Surgery vol. 31 (2016).

35. Kamal, M. M. et al. REST regulates oncogenic properties of glioblastoma stem cells. Stem Cells 30, 405-414 (2012).

36. Ellis, H. P. \& Kurian, K. M. Biological Rationale for the Use of PPAR $\hat{\mathrm{I}}^{3}$ Agonists in Glioblastoma. Front. Oncol. 4, 52 (2014).

37. Yamini, B. NF-kB, Mesenchymal Differentiation and Glioblastoma. Cells 7, 125 (2018).

38. Avci, N. G. et al. NF- $\kappa \mathrm{B}$ inhibitor with Temozolomide results in significant apoptosis in glioblastoma via the NF- $\kappa \mathrm{B}(\mathrm{p} 65)$ and actin cytoskeleton regulatory pathways. Sci. Rep. 10, 13352 (2020).

39. da Hora, C. C. et al. Sustained NF- $\kappa$ B-STAT3 signaling promotes resistance to Smac mimetics in Glioma stem-like cells but creates a vulnerability to EZH2 inhibition. Cell Death Discov. 5, 72 (2019).

40. Debinski, W. \& Gibo, D. M. Fos-related antigen 1 modulates malignant features of glioma cells. Mol. Cancer Res. 3, 237-249 (2005).

41. Debinski, W. et al. VEGF-D is an X-linked/AP-1 regulated putative onco-angiogen in human glioblastoma multiforme. Mol. Med. 7, 598-608 (2001).

42. Azar, W. J., Zivkovic, S., Werther, G. A. \& Russo, V. C. IGFBP-2 nuclear translocation is mediated by a functional NLS sequence and is essential for its pro-tumorigenic actions in cancer cells. Oncogene 33, 578-588 (2014).

43. Yau, S. W., Azar, W. J., Sabin, M. A., Werther, G. A. \& Russo, V. C. IGFBP-2 - taking the lead in growth, metabolism and cancer. J. Cell Commun. Signal. 9, 125-142 (2015).

44. Schütt, B. S., Langkamp, M., Rauschnabel, U., Ranke, M. B. \& Elmlinger, M. W. Integrinmediated action of insulin-like factor binding protein-2 in tumor cells. J. Mol. Endocrinol. 32, 859868 (2004).

45. Holmes, K. M. Elucidating the IGFBP2 signaling pathway in glioma development Elucidating the IGFBP2 signaling pathway in glioma development and progression and progression. https://digitalcommons.library.tmc.edu/utgsbs_dissertations (2012).

46. McDonald, K. L. et al. IQGAP1 and IGFBP2. J. Neuropathol. Exp. Neurol. 66, 405-417 (2007).

47. Azar, W. J. et al. IGFBP-2 enhances VEGF gene promoter activity and consequent promotion of angiogenesis by neuroblastoma cells. Endocrinology 152, 3332-3342 (2011).

48. Adiseshaiah, P., Peddakama, S., Zhang, Q., Kalvakolanu, D. V. \& Reddy, S. P. Mitogen regulated induction of FRA-1 proto-oncogene is controlled by the transcription factors binding to both serum and TPA response elements. Oncogene 24, 4193-4205 (2005).

49. Vial, E. \& Marshall, C. J. Elevated ERK-MAP kinase activity protects the FOS family member FRA-1 against proteasomal degradation in colon carcinoma cells. J. Cell Sci. 116, 4957-4963 (2003).

50. Simpson, A., Petnga, W., Macaulay, V. M., Weyer-Czernilofsky, U. \& Bogenrieder, T. Insulin- 
Like Growth Factor (IGF) Pathway Targeting in Cancer: Role of the IGF Axis and Opportunities for Future Combination Studies. Target. Oncol. 12, 571-597 (2017).

51. Yao, X., Sun, S., Zhou, X., Guo, W. \& Zhang, L. IGF-binding protein 2 is a candidate target of therapeutic potential in cancer. Tumor Biology vol. 37 1451-1459 (2016).

52. Salaroglio, I. C., Mungo, E., Gazzano, E., Kopecka, J. \& Riganti, C. ERK is a pivotal player of chemo-immune-resistance in cancer. International Journal of Molecular Sciences vol. 20 (2019).

53. Abrams, S. L. et al. The Raf/MEK/ERK pathway can govern drug resistance, apoptosis and sensitivity to targeted therapy. Cell Cycle 9, 1781-1791 (2010).

54. Li, T. et al. IGFBP2: integrative hub of developmental and oncogenic signaling network. Oncogene vol. 39 2243-2257 (2020).

55. Phillips, L. M. et al. Glioma progression is mediated by an addiction to aberrant IGFBP2 expression and can be blocked using anti-IGFBP2 strategies. J. Pathol. 239, 355-364 (2016).

56. Gusev, Y. et al. Data descriptor: The REMBRANDT study, a large collection of genomic data from brain cancer patients. Sci. Data 5, (2018).

57. Gautier, L., Cope, L., Bolstad, B. M. \& Irizarry, R. A. affy--analysis of Affymetrix GeneChip data at the probe level. Bioinformatics 20, 307-315 (2004).

58. Leek, J. T., Johnson, W. E., Parker, H. S., Jaffe, A. E. \& Storey, J. D. The SVA package for removing batch effects and other unwanted variation in high-throughput experiments. Bioinformatics 28, 882-883 (2012).

59. Ritchie, M. E. et al. limma powers differential expression analyses for RNA-sequencing and microarray studies. Nucleic Acids Res. 43, e47-e47 (2015).

60. Wingender, E., Dietze, P., Karas, H. \& Knüppel, R. TRANSFAC: A database on transcription factors and their DNA binding sites. Nucleic Acids Research vol. 24 238-241 (1996).

61. Integrating pathway data for systems pathology - PubMed. https://pubmed.ncbi.nlm.nih.gov/17822386/.

62. Aken, B. L. et al. The Ensembl gene annotation system. Database 2016, baw093 (2016).

63. Subramanian, A. et al. Gene set enrichment analysis: A knowledge-based approach for interpreting genome-wide expression profiles. Proc. Natl. Acad. Sci. U. S. A. 102, 15545-15550 (2005).

64. Liberzon, A. et al. Molecular signatures database (MSigDB) 3.0. Bioinformatics 27, 1739-1740 (2011).

65. Yu, G., Wang, L.-G., Yan, G.-R. \& He, Q.-Y. DOSE: an R/Bioconductor package for disease ontology semantic and enrichment analysis. Bioinformatics 31, 608-609 (2015).

66. Chen, E. Y. et al. Enrichr: Interactive and collaborative HTML5 gene list enrichment analysis tool. BMC Bioinformatics 14, (2013).

67. Gravendeel, L. A. M. et al. Intrinsic gene expression profiles of gliomas are a better predictor of survival than histology. Cancer Res. 69, 9065-9072 (2009).

68. Results of the analysis using the pipeline My-Genome-Enhancer in geneXplain platformhttps://platform.genexplain.com/bioumlweb/\#anonymous=true\&de=data/Projects/Regulatory_ networks_of_Glioblastoma_survival/Data/GSE_dataset/Master_regulator_network 
